\title{
Detection of an X-ray Pulsation for the Gamma-ray Pulsar Centered in CTA 1
}

\author{
L. C.C. $\operatorname{Lin}^{1}$, R. H. H. Huang ${ }^{2}$, J. Takata ${ }^{3}$, C. Y. Hwang ${ }^{1}$, A. K. H. Kong ${ }^{2,5}$ and C. Y. Hui ${ }^{4}$ \\ lupin@crab0.astr.nthu.edu.tw
}

\begin{abstract}
We report the detection of X-ray pulsations with a period of $\sim 315.87 \mathrm{~ms}$ from the 2009 $X M M$-Newton observation for the radio-quiet $\gamma$-ray pulsar, LAT PSR J0007+7303, centered in the supernova remnant CTA 1 . The detected pulsed period is consistent with the $\gamma$-ray periodicity at the same epoch found with the Fermi Gamma-ray Space Telescope. The broader sinusoidal structure in the folded light curve of the X-ray emission is dissimilar to that of the $\gamma$-ray emission, and the phase of the peak is about 0.5 shifting from the peak in the $\gamma$-ray bands, indicating that the main component of the X-rays originates from different sites of the pulsar. We conclude that the main component of the X-ray pulsation is contributed by the thermal emission from the neutron star. Although with a significantly different characteristic age, PSR J0007+7303 is similar to Geminga in emission properties of X-rays and $\gamma$-rays; this makes PSR J0007+7303 the second radio-quiet $\gamma$-ray pulsar with detected X-ray pulsations after Geminga.
\end{abstract}

Subject headings: radiation mechanisms: non-thermal — radiation mechanisms: thermal — pulsars: individual (PSR J0007+7303, Geminga) — X-rays: general — gamma rays: general

\section{Introduction}

In the first year of its operation, Fermi observatory detected 23 new radio-quiet $\gamma$-ray pulsars (Abdo et al. 2009; Saz Parkinson et al. 2010). The X-ray counterparts for some of these $\gamma$-ray pulsars have been suggestively proposed, e.g., CXOU J180950.2-233223 (Braie \& Romani 2002), RX J1836.2+5925 (Halpern et al. 2002, 2007), and 2XMM J202131.0+402645 (Trepl et al. 2010); these sources show similar spectral features as a neutron star. Although these pulsars might be claimed as a next Geminga (e.g., Abdo et al. 2010), their pulsed emission in X-ray bands have not been confirmed so far.

\footnotetext{
${ }^{1}$ Graduate Institute of Astronomy, National Central University, Jhongli 32001, Taiwan

${ }^{2}$ Institute of Astronomy and Department of Physics, National Tsing Hua University, Hsinchu 30013, Taiwan

${ }^{3}$ University of Hong Kong, Department of Physics, Hong Kong, PRC

${ }^{4}$ Department of Astronomy and Space Science, Chungnam National University, Daejeon, South Korea

${ }^{5}$ Golden Jade Fellow of Kenda Foundation, Taiwan
}

The X-rays from these radio-quiet $\gamma$-ray pulsars are expected to be generally characterized by thermal emission from the neutron star surface and the hot polar cap as well as non-thermal emission from the magnetosphere and the pulsar wind nebula (Caraveo et al. 2003, 2004). These different emission components can possibly have their contributions in different rotational phases (cf. Caraveo et al. 2004). Therefore, it is not possible to obtain a complete picture of this unique class of pulsars without the detection of X-ray pulsations. Nevertheless, until now, Geminga is still the only one in this class with the X-ray pulsed signals identified.

In the search for X-ray pulsations from $\gamma$ ray-only-emitting pulsars, the point source centered in the composite supernova remnant (SNR) CTA $1(\mathrm{G} 119.5+10.2)$ is one of the most intriguing candidates. CTA 1 was firstly discovered by Owens Valley Radio Observatory in 1960s (Harris \& Roberts 1960); previous studies (Seward et al. 1995) revealed that the X-rays from the center of CTA 1 consist of diffuse emission plus 

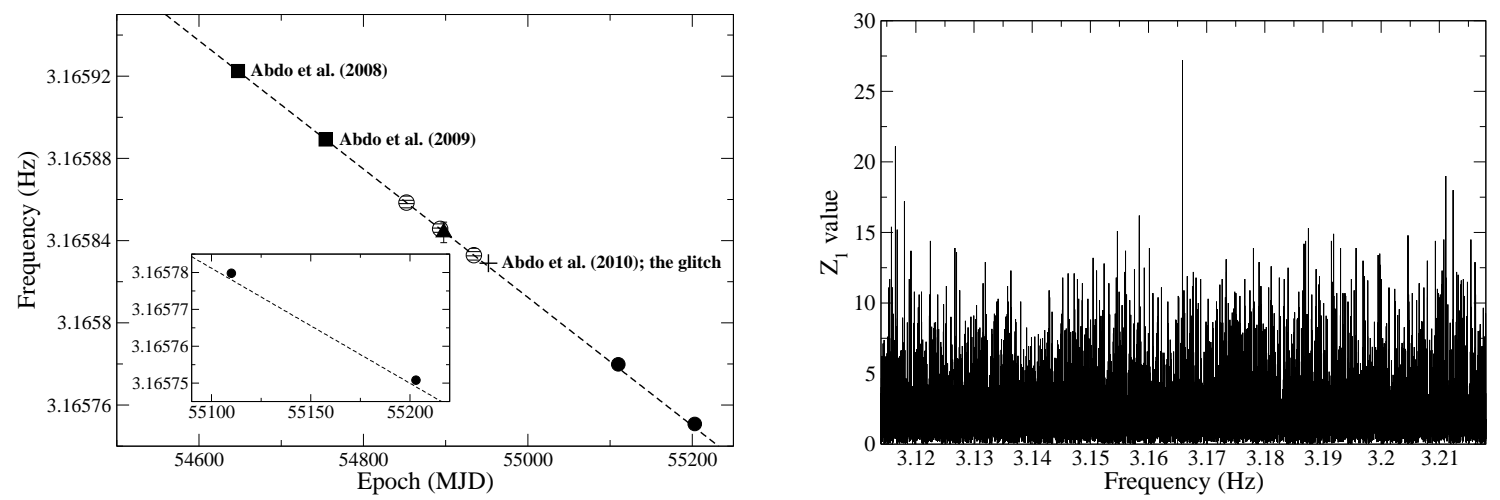

Fig. 1. - Left panel: Spin periods of PSR J0007+7303 from Fermi and XMM. The squares represent the spin periods detected by Abdo et al. (2008, 2009). The plus sign marks the glitch of PSR J0007+7303 with df $/ \mathrm{f}=5 \times 10^{-7}$ Abdo \& Fermi-LAT Collaboration 2010). The circles represent the spin periodicities detected by the Fermi data before the glitch, and the solid circles represent those after the glitch. The triangle represents the period detected by the $X M M$ data of 2009 with an uncertainty shown by the error bar. The dashed line represents the period derivative $-3.6133 \times 10^{12} \mathrm{~Hz} / \mathrm{s}$ derived by Abdo et al. (2009). The periods after glitch have a little shift from the dashed line which are clearly shown in the insert panel. Right panel: Rayleigh test of XMM data around the frequency $[3.114,3.218] \mathrm{Hz}$. The width of each independent trial is about $1.037 \times 10^{-5} \mathrm{~Hz}$. The most significant signal appears at the frequency of $3.165844 \mathrm{~Hz}$.

a faint point source RX J0007.0+7302. The precise position of RX J0007.0+7302 was provided by the Chandra image (Halpern et al. 2004). No radio counterpart has been found at the position of RX J0007.0+7302 (Pineault et al. 1993). On the other hand, the high $\gamma$-ray-to-X-ray and X-ray-to-optical flux ratios (Brazier et al. 1998; Halpern et al. 2004) and the morphology of a bent jet from a torus-like pulsar wind nebula (PWN) revealed in X-ray image (Halpern et al. 2004) all characterize RX J0007.0+7302 as a neutron star. Further studies of the spectral properties for RX J0007.0+7302 were also consistent with a pulsar without radio emission, e.g., Geminga (Slane et al. 2004). In addition, based on the positional coincidence and the emission properties, RX J0007.0+7302 was also identified as the Xray counterpart of $3 \mathrm{EG} \mathrm{J} 0010+7309$, which is a $\gamma$-ray source found by the Energetic Gamma-Ray Experiment Telescope (EGRET) on the Compton Gamma-Ray Observatory (Slane et al. 1997; Brazier et al. 1998).

Although all these observations suggested that RX J0007.0+7302 is likely to be a radio-quiet $\gamma$-ray pulsar associated with the supernova remnant (Slane et al. 1997), the final confirmation came from the detection of $\gamma$ pulsations with Fermi (Abdo et al. 2008). The detected $\gamma$-ray pulsation has a spin period of $315.86 \mathrm{~ms}$. This pulsar has a characteristic spin-down age of $\tau \sim 14000 \mathrm{yr}$, which is consistent with the age of CTA 1 (10000 - 20000 yr, Zhang \& Cheng 1998 and Slane et al. 2004). This further supports the scenario that this pulsar is indeed associated with CTA 1. In addition, a precise position of LAT PSR J0007+7303 can be inferred from the Chandra image (Halpern et al. 2004; Abdo et al. 2009). However, the search of the corresponding pulsed signal in the archival Xray data obtained before 2009 was unsuccessful. Caraveo (2009) indicated that the X-ray pulsation can be derived from the contemporaneous Fermi ephemeris of PSR J0007+7303 although no any further information of the X-ray pulsation was provided. This leaves the X-ray temporal properties of RX J0007.0+7303 still unconstrained.

In this Letter, we report the detection of X-ray pulsations from PSR J0007+7303 using the 2009 $X M M-N e w t o n$ (hereafter XMM) observation (PI: Caraveo 2008, ObsID: 06049401). We show detailed information of temporal and spectral analyses for this source. We investigate the source spectrum and the folded light curves in different energy bands to determine the possible origins of the $\mathrm{X}$ ray emission from PSR J0007+7303. 


\section{Observations and data analysis}

The central region of CTA 1 was observed by $X M M$ twice with an epoch separation of $\sim 7$ years. The first observation was performed on 2002 February 21 for $\sim 40$ ks and the second one was carried out on 2009 March 7 for $\sim 120$ ks. These two observations were operated in the fullframe mode for the two MOS detectors and in the small-window mode for the pn detector. The medium and thin filters were used for the two MOS detectors and the pn detector, respectively. The temporal resolution of $\sim 6 \mathrm{~ms}$ for the small-window mode of pn detector is suitable for the periodicity search of X-ray pulses from PSR J0007+7303. Slane et al. (2004) have already analyzed the old $X M M$ data observed on 2002 February 21 but no significant pulsation was detected using a total of 1055 counts in the energy band $0.3-10 \mathrm{keV}$ and an upper limit of $61 \%$ for the pulsed fraction was reported with a sinusoidal profile. In our study, we focus on the 2009 XMM data. All the data were processed with the SAS version of 10.0.0.

\subsection{Timing analysis}

We first performed the source detection by using the task "edetect_chain" and determined the position of PSR J0007+7303 in the X-ray band at (J2000) R.A. $=00^{h} 07^{m} 01^{s} .2$, Dec. $=+73^{\circ} 03^{\prime} 07^{\prime \prime} .6$ with an uncertainty of $\sim 0^{\prime \prime} .6$. The point source was then extracted at this position within a circular region of $15^{\prime \prime}$ in radius, which is consistent with the $70 \%$ of the encircled energy function. After screening the background flare, the effective exposure is $\sim 67 \mathrm{ks}$. Following the standard process of the data reduction, 2989 counts were yielded in the energy band $0.2-12 \mathrm{keV}$. Furthermore, the photon arrival times were corrected to the solar system barycenter with the "barycen" task (JPL DE200 earth ephemeris) of the SAS package.

Abdo et al. (2009) reported a periodic signal of $3.1658891845(5) \mathrm{Hz}$ at the reference epoch MJD 54754 with a frequency derivative of $-3.6133(3) \times$ $10^{-12} \mathrm{~Hz} / \mathrm{s}$ for PSR J0007+7303. According to this timing ephemeris, a periodicity of $3.165844344(4) \mathrm{Hz}$ is expected at the start of the GTI of the 2009 XMM data (epoch MJD 54897.6335129). We thus search for periodic signals with the known frequency derivative around the predicted frequency in the X-ray data. By

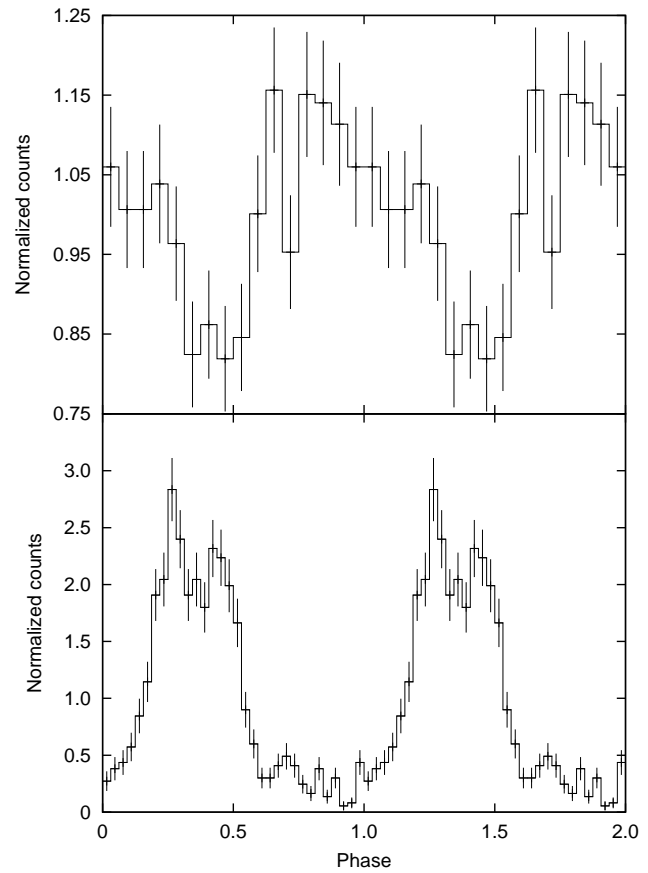

Fig. 2. - Folded light curves of PSR J0007+7303. The upper panel shows the pulse profile in the $0.2-12$ $\mathrm{keV}$ from the $2009 X M M$ observation and the lower one shows the profile in the $100 \mathrm{MeV}$ to $300 \mathrm{GeV}$ from the Fermi observations. The counts were normalized to the average photons of each bin. All the curves were folded simultaneously with the periodic frequency and the frequency derivative of the same epoch at the start of GTI of the $2009 X M M$ data as we described in the main text.

performing an $H$-test de Jager et al. 1989), we found a test statistic of $H \sim 27.0$, which corresponds to a random probability of $2.0 \times 10^{-5}$ for a single trial (de Jager \& Büsching 2010). The uncertainty of our detection was determined by the equation provided by Leahy (1987) and the most significant trial for the periodicity search was determined at $3.165844(5) \mathrm{Hz}$ as shown in the left panel of Fig. 1. The $H$-statistic also obtains the most significant periodic detection with a sinusoidal structure consisting of only one harmonic. We then verified the $Z_{1}$ value of 10000 independent nearby trials $(\sim 3.114 \mathrm{~Hz}-3.218 \mathrm{~Hz})$ by a Rayleigh test. Our detection of the periodic signal is highly significant as shown in the right panel of Fig. 1 .

In order to compare the pulse profiles in X-rays 
and in $\gamma$-rays, we obtain $\sim 3$-month Fermi data (2009 January 21 - 2009 April 13) within the energy range of $100 \mathrm{MeV}$ to $300 \mathrm{GeV}$ on the epochs close to the 2009 XMM observation. We selected a 1-degree radius region centered on the source at R.A. $=1.7565^{\circ}$, Dec. $=73.0523^{\circ}(\mathrm{J} 2000)$ according to the position of the X-ray counterpart detected by Chandra. The pulse profiles folded simultaneously with the frequency of 3.165844344 $\mathrm{Hz}$ and the frequency derivative of $-3.6133(3) \times$ $10^{-12} \mathrm{~Hz} / \mathrm{s}$ at epoch MJD 54897.6335129 for the $X M M$ and the Fermi observations are shown in Fig. 2. The pulse profile in the X-ray band shows a large pulsed phase $(0.5625-1.3125)$ with 0.75 duty cycle while in the $\gamma$-ray band the pulse profile shows a small pulsed phase $(0.125-0.5625)$ with $\sim 0.45$ duty cycle. In addition, the peaks of these two light curves shift about 0.5 phase, indicating that the $\mathrm{X}$-rays and $\gamma$-rays originate from different sites of the pulsar.

\subsection{Spectral analysis}

The EPIC spectra of PSR J0007+7303 were extracted using the aforementioned 15"-radius source region. The background was defined as an annulus with the inner and outer radii of $75^{\prime \prime}$ and $120^{\prime \prime}$ centered at the source region. The photon redistribution matrices and the ancillary region files were generated by the task "rmfgen" and "arfgen" of the SAS package. In order to compare with the previous analysis reported by Slane et al. (2004); Halpern et al. (2004), our spectra were restricted in the energy range $0.5-10.0 \mathrm{keV}$ and each spectral bins were rebinned with a minimum of 30 counts. To reduce the uncertainties in spectral fitting, we set the column density to be $\sim 2.8 \times 10^{21} \mathrm{~cm}^{-2}$ according to the previous ASCA/ROSAT measurements of CTA 1 (Slane et al. 1997). This value is also consistent with the optical extinction (Halpern et al. 2004).

We have examined the spectrum by fitting with various single component models that include power-law (PL), blackbody(BB) and hydrogen atmospheric model of a neutron star (NSA; Zavlin et al. 1996). We find that no single component can fit the spectrum well. Instead, the spectrum can be described by a composite model consisting of a non-thermal component and a thermal component. We summarize the best-fit spectral parameters in Table [1] The non-thermal X-
Table 1: Best-fit spectral parameters for PSR J0007+7303.

\begin{tabular}{l|cc}
\hline Parameter & PL+BBodyrad & PL+NSA \\
\hline$N_{H}\left(\mathrm{~cm}^{-2}\right)$ & $2.8 \times 10^{21}$ (fixed) & $2.8 \times 10^{21}($ fixed $)$ \\
$\Gamma$ & $1.52_{-0.09}^{+0.10}$ & $1.49_{-0.11}^{+0.10}$ \\
$F_{X, \mathrm{PL}}\left(\mathrm{ergs} \mathrm{cm}^{-2} \mathrm{~s}^{-1}\right)^{a}$ & $1.6 \times 10^{-13}$ & $1.6 \times 10^{-13}$ \\
$k T(\mathrm{keV})$ & $0.104 \pm 0.013$ & $0.058_{-0.011}^{+0.019}$ \\
$F_{X, \mathrm{Th}}\left(\mathrm{ergs} \mathrm{cm} \mathrm{cm}^{-2} \mathrm{~s}^{-1}\right)^{a}$ & $3.3 \times 10^{-14}$ & $3.5 \times 10^{-14}$ \\
$R(\mathrm{~km})^{b}$ & $1.39_{-0.43}^{+0.68}$ & $10($ fixed $)$ \\
$\chi^{2} /$ dof & $108.9 / 119$ & $108.0 / 119$ \\
\hline Note: Quoted errors indicate the $90 \%$ confidence level for \\
one parameter of interest. \\
$a$ The unabsorbed flux is measured in the range of $0.5-10$ \\
keV. \\
$b$ The radius is measured from the normalization factor \\
for a source distance of 1.4 kpc. \\
$c$ The NSA model is proposed to be nonmagnetic with the \\
mass and radius of the neutron star fixed at $1.4 \mathrm{M} \odot$ and \\
10 km.
\end{tabular}

ray emission from the pulsar PSR J0007+7303 is thought to come from the outer magnetospheric gap (Takata et al. 2006, 2008). The thermal contribution with a temperature of $\sim 0.6-1.0 \times 10^{6} \mathrm{~K}$ might originate from the surface of the neutron star. The X-ray flux from the thermal component is only $\sim 20 \%$ of the total flux, which is consistent with previous $X M M$ detection (Slane et al. 2004). However, the observed $X M M$ flux is $\sim 4$ times of the flux attributed to the pulsar in the Chandra observation (Halpern et al. 2004). We note that the aperture radius of $X M M$ is about 15 ", which is much larger than the point-spread-function of Chandra and might contain emission from the PWN or/and the jet. The excess of the thermal flux detected by $X M M$ data may be also due to the thermal emission contributed from the diffuse emission of CTA 1 (Slane et al. 1997). We also note that the Chandra spectrum was fitted with only 6 dofs, suggesting the model fitting might have large uncertainty. In this respect, the uncertainty of the thermal flux derived by the Chandra spectrum might be large. On the other hand, although the resolving power of XMM-Newton is worse, the larger effective area of the detectors onboard XMM-Newton and the longer exposures of the $2009 X M M$ data should provide much better photon statistics than that of the Chandra data. Furthermore, we note that the X-ray light curve shows that the pulsed components can be as large as $\sim 20 \%$ of the DC level, indicating that the soft X-ray flux from the pulsar could be at least as large as $\sim 20 \%$ of the detected flux even the ob- 
Fig. 3. - Broad band pulsed spectrum of PSR J0007+7303.

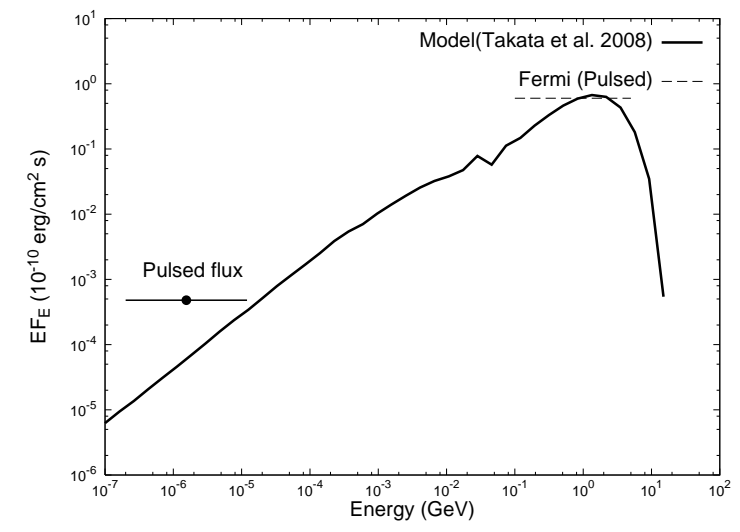

served total flux might be contaminated by PWN or jets around the pulsar. We also note that the sum of the individual non-thermal contributions from the jet, PWN and the pulsar reported by Halpern et al. (2004) is fully consistent with that inferred from the $X M M$ data, though it is not possible to disentangle various components with this data set. These indicate that the fluxes of these two data sets are not inconsistent.

\section{Discussion}

An X-ray pulsation was detected using 2009 $X M M-N e w t o n$ data. However, it is difficult to obtain a pulsed spectrum with present data. The photons of the pulsed component are quite few with the pulsed fraction $\sim(17 \pm 1) \%$ of the total $\mathrm{X}$-ray flux. In addition, the EPIC background is dominated by thermal emission at lower energies $(\mathrm{E}<1 \mathrm{keV})$, which causes large uncertainties in the pulsed spectrum. For $\gamma$-ray pulsars in general, however, the X-ray emission can be fitted by a thermal component from the neutron star surface and/or a non-thermal component from the relativistic particles that also emit the $\gamma$-rays. It has been observed from several $\gamma$-ray pulsars (e.g., Geminga and Vela) that the peak in X-ray light curve of the non-thermal component is in phase with the $\gamma$-ray peak, while the peak phase of the thermal component is different from that in the $\gamma$-ray bands. The $\sim 0.5$ phase shift between the peaks of the folded light curves in the X-ray and the $\gamma$-ray bands, as shown in Fig. 2 , indicates that the main pulsed component of the X-rays prob-

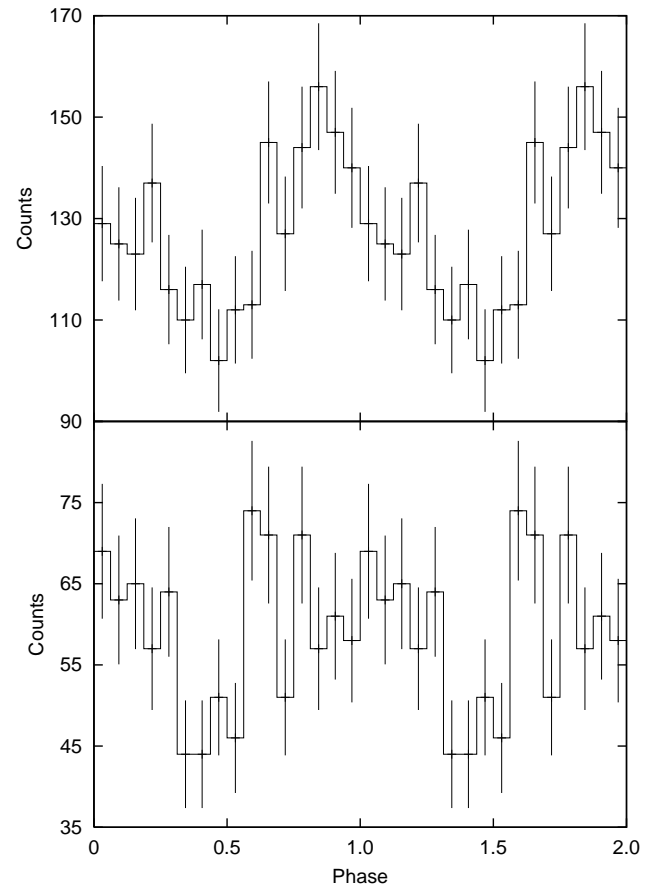

Fig. 4. - Folded light curves of PSR J0007+7303 in the $0.2-2 \mathrm{keV}$ (upper panel) and in the $2-12 \mathrm{keV}$ (lower panel). These two curves were folded with the frequency of $3.165844344 \mathrm{~Hz}$ and the frequency derivative of $-3.6133 \times 10^{-12} \mathrm{~Hz} / \mathrm{s}$ simultaneously at the same epoch zero of MJD 54897.6335129.

ably originates from the thermal emission of the neutron star.

In Fig. 3. we compare the observed pulsed spectrum with the theoretical predictions of the outer gap accelerator model investigated by Takata et al. (2008), where the $\gamma$-rays and the $\mathrm{X}$-rays are emitted via curvature radiation and synchrotron radiation of the relativistic particles in the magnetosphere, respectively. We can see from Fig. 3 that the observed pulsed flux integrated from 0.2 to $12 \mathrm{keV}$ of $\sim(5 \pm 0.2) \times$ $10^{-5}$ photons $\mathrm{cm}^{-2} \mathrm{~s}^{-1}$ is much larger than the predicted pulsed flux $\left(\sim 10^{-5}\right.$ photons $\left.\mathrm{cm}^{-2} \mathrm{~s}^{-1}\right)$ of the non-thermal component, suggesting the observed pulsed component is not dominated by the non-thermal emission from the outer gap accelerator.

In order to examine the energy dependence of the pulsed X-ray emission from PSR J0007+7303, we divided the source photons into the soft X-ray 
$(0.2-2 \mathrm{keV})$ and hard X-ray $(2-12 \mathrm{keV})$ band. Fig. 4 shows the folded light curves in the soft (upper panel) and in the hard (lower panel) X-ray bands. For the soft X-ray energy band, the random probability to yield the pulsed detection is $3.1 \times 10^{-5}\left(Z_{1}^{2}=20.8\right)$, and the pulse profile has a broad sinusoidal structure, which shows a similar X-ray pulse profile as that in Fig. 2. In the hard X-ray band, on the other hand, the random probability to yield the pulsed detection is only $0.039\left(Z_{1}^{2}=6.49\right)$, and the structure of the pulse profile is much more random. We did not detect pulsed signals at hard X-rays. This suggests that the X-ray pulsation detected for PSR J0007+7303 is mainly from the thermal emission. This is also consistent with the fact that the thermal X-ray emission from a neutron star, which may not contribute significantly to the hard X-rays $(>2 \mathrm{keV})$. Without a firm detection of pulsed emission in the hard X-ray band, the nature of the power-law component remains unclear.

One can use some physical properties of pulsars such as ages and spin-down powers to characterize features of $\gamma$-ray pulsars. Based on these physical properties, PSR J0007+7303 would be considered to be similar to a Vela-like pulsar. On the other hand, one can also consider the similarity between two pulsars by comparing their high-energy emission properties. In this respect, PSR J0007+7303 has the certain X-ray pulsed detection and shares the similar spectral features with the Geminga pulsar; this indicates that the high-energy emission from these two pulsars may have similar mechanisms and makes PSR J0007+7303 as the second Geminga. Both the spectra of PSR J0007+7303 and Geminga show a steepening at the range of $\mathrm{GeV}$ and can be described as a hard power-law with $\Gamma \sim 1.5-$ 1.6 Maver-Hasselwander et al. 1994; Abdo et al. 2008). The X-ray source spectra of these two pulsars can both be fitted by a composite model consisting of a power-law and a blackbody component (Halpern \& Wang 1997; Slane et al. 2004).

However, in contrast to the Geminga pulsar, the X-ray spectral behavior of PSR J0007+7303 is dominated by non-thermal contribution, which might come from the PWN (Slane et al. 1997). Since the pulse profile of the pulsar centered in CTA 1 presents a broad sinusoidal structure as that of the Geminga pulsar (Caraveo et al. 2004), the pulsation in the soft X-ray band is expected to be thermal-dominant. Both sources show significantly dissimilar pulsed profiles for the soft X-ray and the $\gamma$-ray bands (Halpern \& Ruderman 1993 $\&$ this work). In fact, except for the Crab pulsar, most of the EGRET pulsars do not show resemblance for the pulse profiles in the soft X-ray and the $\gamma$-ray bands (Thompson 2001). The hard Xray and the $\gamma$-ray pulsed profiles of the Geminga pulsar have similar features (Kargaltsev et al. 2005), indicating the emission are likely originated from the same locations.

The authors appreciate an anonymous referee for his/her fruitful comments. We also thank Dr. Jau-Shian Liang for discussion on the application of the pulsed spectrum for our target. This work was partially supported by the National Science Council through grant NSC 99-2811-M008-057, and RHHH is supported through grant NSC 099-2811-M-007-062. CY Hwang acknowledges support from the National Science Council through grants NSC 99-2112-M-008-014-MY3 and NSC 99-2119-M-008-017. CY Hui is supported by the research fund of Chungnam National University in 2010. AKHK acknowledges support from the National Science Council through grants NSC 96-2112-M-007-037-MY3 and NSC 99-2112M-007-004-MY3.

Facilities: Fermi, XMM-Newton.

\section{REFERENCES}

Abdo, A., \& Fermi-LAT Collaboration. 2010, in Bulletin of the American Astronomical Society, Vol. 41, Bulletin of the American Astronomical Society, 680-

Abdo, A. A., et al. 2008, Science, 322, 1218

—. 2009, Science, 325,840

—. 2010, ApJ, 712, 1209

Braje, T. M., \& Romani, R. W. 2002, ApJ, 580, 1043

Brazier, K. T. S., Reimer, O., Kanbach, G., \& Carraminana, A. 1998, MNRAS, 295, 819

Caraveo, P. 2008, in XMM-Newton Proposal ID \#06049401, 136 
Caraveo, P. A. 2009, ArXiv e-prints

Caraveo, P. A., Bignami, G. F., De Luca, A., Mereghetti, S., Pellizzoni, A., Mignani, R., Tur, A., \& Becker, W. 2003, Science, 301, 1345

Caraveo, P. A., De Luca, A., Mereghetti, S., Pellizzoni, A., \& Bignami, G. F. 2004, Science, 305, 376

de Jager, O. C., \& Büsching, I. 2010, A\&A, 517, L9

de Jager, O. C., Raubenheimer, B. C., \& Swanepoel, J. W. H. 1989, A\&A, 221, 180

Halpern, J. P., Camilo, F., \& Gotthelf, E. V. 2007, ApJ, 668, 1154

Halpern, J. P., Gotthelf, E. V., Camilo, F., Helfand, D. J., \& Ransom, S. M. 2004, ApJ, 612,398

Halpern, J. P., Gotthelf, E. V., Mirabal, N., \& Camilo, F. 2002, ApJ, 573, L41

Halpern, J. P., \& Ruderman, M. 1993, ApJ, 415, 286

Halpern, J. P., \& Wang, F. Y.-H. 1997, ApJ, 477, 905

Harris, D. E., \& Roberts, J. A. 1960, PASP, 72, 237

Kargaltsev, O. Y., Pavlov, G. G., Zavlin, V. E., \& Romani, R. W. 2005, ApJ, 625, 307

Leahy, D. A. 1987, A\&A, 180, 275

Mayer-Hasselwander, H. A., et al. 1994, ApJ, 421, 276

Pineault, S., Landecker, T. L., Madore, B., \& Gaumont-Guay, S. 1993, AJ, 105, 1060

Saz Parkinson, P. M., et al. 2010, ArXiv e-prints

Seward, F. D., Schmidt, B., \& Slane, P. 1995, ApJ, 453,284

Slane, P., Seward, F. D., Bandiera, R., Torii, K., \& Tsunemi, H. 1997, ApJ, 485, 221

Slane, P., Zimmerman, E. R., Hughes, J. P., Seward, F. D., Gaensler, B. M., \& Clarke, M. J. 2004, ApJ, 601, 1045
Takata, J., Chang, H.-K., \& Shibata, S. 2008, MNRAS, 434

Takata, J., Shibata, S., Hirotani, K., \& Chang, H.-K. 2006, MNRAS, 366, 1310

Thompson, D. J. 2001, in American Institute of Physics Conference Series, Vol. 558, American Institute of Physics Conference Series, ed. F. A. Aharonian \& H. J. Völk, 103

Trepl, L., Hui, C. Y., Cheng, K. S., Takata, J., Wang, Y., Liu, Z. Y., \& Wang, N. 2010, MNRAS, 405, 1339

Zavlin, V. E., Pavlov, G. G., \& Shibanov, Y. A. 1996, A\&A, 315, 141

Zhang, L., \& Cheng, K. S. 1998, A\&A, 335, 234

This 2-column preprint was prepared with the AAS IATEX macros v5.2. 